\title{
EXTRACTOS VEGETALES \\ PARA EL CONTROL IN VITRO \\ DE COLLETOTRICHUM \\ GLOEOSPORIOIDES P. AISLADO DE \\ CARICA PAPAYA L
}

\author{
PLANT EXTRACTS FOR THE IN VITRO CONTROL OF \\ COLLETOTRICHUM GLOEOSPORIOIDES P. ISOLATED FROM \\ CARICA PAPAYA L.
}

\author{
Tatiana Paola Duarte Rodríguez ${ }^{1}$ \\ Sandra Isabel Ramírez González ${ }^{2}$ \\ sandra.ramirez@unach.mx \\ Orlando López Báez ${ }^{2}$ \\ olopez@unach.mx \\ Ana Cruz Morillo Coronado ${ }^{1}$ \\ ana.morillo@uptc.edu.co
}

1 Universidad Pedagógica y Tecnológica de Colombia, Colombia

2 Universidad Autónoma de Chiapas, México 
Para citar este artículo:

Duarte Rodríguez, T. P., Ramírez González, S. I., López Báez, O., \& Morillo Coronado, A. C. (2021). Extractos vegetales para el control in vitro de Colletotrichum gloeosporioides $P$. aislado de Carica papaya L. ESPACIO I+D, INNOVACIÓN MÁS DESARROLLO, 10(26). https:// doi.org/10.31644/IMASD.26.2021.a05

\section{RESUMEN}

La papaya es un cultivo de gran importancia a nivel mundial. México y Colombia son líderes mundiales en su producción; una de las principales limitantes para este cultivo son las enfermedades dentro de las que destaca la antracnosis que afecta en pre y poscosecha, es por ello que la investigación en la búsqueda de alternativas eficientes, sustentables e inocuas en la sanidad del cultivo son primordiales para reducir las pérdidas en la producción y con ello mejoren los ingresos y la calidad de vida del productor y los involucrados en la cadena productiva, hasta el consumidor, con productos de calidad y sin contaminantes que afecten su salud. El objetivo de este estudio fue evaluar extractos de plantas bajo dos formas de extracción en el control in vitro de Colletotrichum gloesporioides. Por lo que fue evaluado el efecto antifúngico de extractos vegetales de hojas de orégano (Oreganum vulgare), frutos de pimienta (Pimienta dioica), hojas de neem (Azadirachta indica.) y hojas secas de matarratón (Gliricidia sepium) obtenidos a partir de métodos de destilación y microondas, sobre C. gloesporioides aislado de Carica papaya. Fue utilizada la técnica de medio envenenado en cajas Petri con medio PDA; en una primera fase, se probó la concentración $60 \%$ para todos los extractos. Posteriormente fue determinada la concentración mínima inhibitoria para aquellos que en la primera fase inhibieron el $100 \%$. El efecto antifúngico se determinó mediante crecimiento micelial, número de conidias totales y germinadas. Los resultados indicaron que el extracto de fruto de pimienta obtenido por microondas, en una concentración de $40 \%$ y el producto patente M2 UNACH en una concentración de $20 \%$, inhibieron totalmente a C. gloesporioides. los extractos obtenidos mediante el microondas tuvieron mejor efecto antifúngico que los obtenidos por destilación.

\section{Palabras clave}

Antracnosis; Pimienta dioica; microondas; antifúngico. 
Papaya is a crop of great importance worldwide, Mexico and Colombia are world leaders in its production; one of the main limitations for this crop is the diseases that include the anthracnose that affects pre and post harvest, which is why research in the search for efficient, sustainable and harmless alternatives in crop health are essential to reduce losses in production and thereby improve the income and quality of life of the producer and those involved in the production chain to the consumer with quality products and without contaminants that affect their health. The objective of this study was to evaluate plant extracts under two forms of extraction in the in vitro control of Colletotrichum gloesporioides. Therefore, the antifungal effect of plant extracts of oregano leaves (Oreganum vulgare), pepper fruits (Pimienta dioica), neem leaves (Azadirachta indica) and dried marathon leaves (Gliricidia sepium) obtained from methods was evaluated: distillation and microwave, on C. gloesporioides isolated from Carica papaya. The technique of poisoned medium in Petri dishes with PDA medium was used, in a first phase the concentration of $60 \%$ was tested for all extracts. Subsequently, the minimum inhibitory concentration was determined for those who in the first phase inhibited $100 \%$. The antifungal effect was determined by mycelial growth, number of total and germinated conidia. The results indicated that the extract of pepper fruit obtained by microwave in a concentration of $40 \%$ and M2 UNACH patent of $20 \%$ inhibited C. gloesporioides t. the extracts obtained by the microwave had better antifungal effect than those obtained by distillation.

\section{Keywords}

Anthracnose; Pimienta dioica; microwave; antifungal. 
L a papaya es también llamada lechosa en Venezuela, fruta bomba en Cuba y Mamao en Brasil. Es una de las frutas tropicales más apetecidas por su suave y agradable sabor y las propiedades nutritivas que se le atribuyen. Es muy solicitada por los consumidores que tienen preferencia por las frutas que además de agradables posean un contenido alto de nutrientes (Corporación Colombiana de Investigación Agropecuaria [Corpoica], 2000). La producción de papaya (Carica papaya L.) tiene gran importancia económica, México ocupa el quinto lugar como productor mundial y el primer lugar como país exportador, a su vez Colombia se encuentra en el octavo lugar como productor mundial (Evans, 2015). En Colombia se presenta un volumen de producción relevante para el cultivo de papaya, según la Encuesta Nacional Agropecuaria (ENA) publicada por el Departamento Administrativo Nacional de Estadística (DANE, 2016), durante el año 2015 en Colombia se obtuvo una producción de 105.459 toneladas de papaya. El cultivo de la papaya está sujeto a diversas enfermedades durante los periodos lluviosos; de estas son consideradas las de mayor importancia: la mancha anular de la papaya, seguida por la antracnosis, la pudrición de raíz y del pie y la mancha o peca negra (DANE, 2016). Colletotrichum gloeosporioides P., es el agente patógeno causante de la antracnosis en Carica papaya L., enfermedad que se encuentra en todas las áreas donde se cultiva papaya y llega a ser la causa principal de las pérdidas de fruta en poscosecha (Instituto de Investigaciones Agropecuarias [INIA], 2016), se estiman pérdidas del 25 al 40\% (Quiroga, 2016) y se presenta en todas las regiones productoras del mundo, causando enormes pérdidas en Brasil, Hawaii (USA) y México (Ventura, et al., 2004).

Colletotrichum gloeosporioides (Penz.) Sacc. es un patógeno ubicuo, prolífero y económicamente importante, ya que induce pérdidas sustanciales en el rendimiento, al afectar partes vegetativas al causar deterioro poscosecha de frutos de clima templado, subtropical y tropical. Además de afectar a los frutos, el hongo patógeno igualmente ataca otras partes de la planta como las raíces, las flores y los tallos (DANE, 2016). Una vez en el fruto las esporas del hongo germinan, después de 48 horas forman un tubo germinativo que penetra de manera directa la cutícula del fruto inmaduro, donde permanece latente, pero se reactiva la invasión durante la maduración del fruto (Zavala et al., 2011). Una de las características destacadas de Colletotrichum sp. es su capacidad de sobrevivir en estado de dormancia o quiescencia cuando las condiciones ambientales o fisiológicas del hospedero le impiden desarrollarse (Parra, 2008), el hongo produce un apresorio que penetra el fruto degradando la cutícula y produciendo una hifa subcutícular latente que no se desarrolla hasta que el fruto madura. El patógeno es activado en condiciones con prevalencia de temporadas lluviosas o con humedad relativa alta (Rodríguez et al., 2009). Ha sido considerado como uno de los principales patógenos que ataca las plantaciones 
de frutales durante prefloración, la enfermedad permanece asintomática hasta que el grado de maduración del fruto es tal que el patógeno realiza una invasión intercelular y la enfermedad se expresa, conociéndose dicho fenómeno como infección quiescente (Montaño y Lemus, 2015). Para el control de la antracnosis se deben establecer medidas de manejo integral del cultivo, basadas en acciones preventivas y curativas, orientadas tanto en la etapa de campo como en la de poscosecha (DANE, 2016). El control químico es el más común debido a la importancia económica del cultivo. Se disponen de muchos químicos, entre los más utilizados se encuentran compuestos como: dithiocarbamato, benzamidazoles y triazoles; y otros fungicidas como chlorothalonil, imazalil y prochloraz (Parra, 2008).

Según Dirzo (2008) "El mundo vegetal está cargado de una sorprendente diversidad de metabolitos, a la mayoría de los cuales no se le ha podido definir ninguna participación evidente en los procesos metabólicos primarios de la planta (fotosíntesis, respiración, etc.). Estos compuestos, por lo tanto, se hicieron acreedores del nombre de "metabolitos secundarios". Se estima que más de 100.000 son producidos por las plantas (Pérez y Jiménez, 2011). Los compuestos secundarios no tienen una función aparente en el metabolismo primario pero sí tienen una implicación ecológica como defensa contra herbívoros, virus, hongos, bacterias, así como sustancias alelopáticas (Echeverría, 2012). Las plantas producen compuestos con propiedades antimicrobianas que pueden ser empleadas para controlar diferentes enfermedades en productos hortofrutícolas. La obtención de los extractos vegetales y el estudio de sus compuestos activos propician su empleo contra diferentes fitopatógenos. En condiciones in vitro los extractos inhiben el crecimiento del patógeno, así como la esporulación y germinación de esporas, de modo que ayudan a controlar las enfermedades de frutos y hortalizas (Hernández et al., 2007).

La obtención de extractos vegetales se puede realizar a partir de diferentes métodos, los cuales permiten la separación o purificación de múltiples sustancias, algunos comúnmente utilizados son la destilación que es uno de los métodos más antiguos y más usados para separar y purificar líquidos; el método consiste en aplicar calor a una mezcla de sustancias hasta que una de ellas pasa a la fase de vapor, seguidamente el vapor se enfría al pasar por un refrigerante donde condensa y el líquido se recoge en un recipiente adecuado. En el recipiente inicial quedarán las sustancias menos volátiles o las impurezas (Quercuslab, 2015). También está la tecnología de microondas, que se ha convertido durante los últimos años en una herramienta que mejora la productividad de los procesos, trabaja con radiaciones electromagnéticas que se encuentran en el rango de 0.3 a $300 \mathrm{GHz}(\lambda=1$ hasta $0.001 \mathrm{~m})$ (Martínez et al., 2010). Recientemente el método asistido por microondas ha sido utilizado como método alterno de extracción a escala de laboratorio 
y ha representado grandes ventajas como el ahorro energético, tiempos cortos de proceso, mayor rendimiento, procesos económicos y amigables con el medio ambiente (Puertas, et al., 2013).

El objetivo de este trabajo de investigación fue determinar la efectividad del uso de extractos vegetales en el control in vitro del patógeno causante de antracnosis, con el fin de ofrecer una alternativa sostenible a este problema.

\section{METODOLOGÍA}

La investigación fue realizada en el Laboratorio de Agrotecnologías de la Agencia Universitaria para el Desarrollo (Audes) Cacao-Chocolate de la Universidad Autónoma de Chiapas, ubicado en Ciudad Universitaria, Tuxtla Gutiérrez, estado de Chiapas, México.

La presente investigación fue desarrollada en dos fases: la primera en donde se evaluó la efectividad antifúngica de extractos en una concentración al 60 \% sobre C. gloeosporioides P.; la segunda que correspondió a la determinación de la concentración mínima inhibitoria (СмI) de extractos sobre C. gloeosporioides. En cada fase se trabajó con una cepa de Colletotrichum gloeosporioides P. aislada de frutos de papaya (Carica papaya L.). suministrada por el Laboratorio de Agrotecnologías de la Audes Cacao-Chocolate de la Universidad Autónoma de Chiapas, la cual fue aislada de frutos de C. papaya obtenidos directamente en campo en el municipio de Villaflores, Chiapas en enero de 2018 y fue realizada la multiplicación del patógeno en este mismo laboratorio mediante repiques en medio de cultivo papa dextrosa agar (PDA), los cuales se incubaron a una temperatura de $23^{\circ} \mathrm{C} \pm 2{ }^{\circ} \mathrm{C}$ durante 12 días.

La investigación se realizó mediante un Diseño Completamente al Azar (DCA), iniciando en la fase uno con 11 tratamientos, cada uno con cuatro repeticiones. Los tratamientos fueron distribuidos en ocho extractos vegetales y tres testigos o controles: uno negativo (medio PDA o testigo absoluto) y dos positivos; el fungicida de síntesis química (I.A. Ciprodinil $37,5 \%+$ Fludioxonil 25\%) y un fungicida en proceso de patente (M2 UNACH). En la fase dos se evaluó el extracto de fruto de pimienta obtenido por microondas y el producto M2 UNACH.

La unidad experimental (UE) en la fase I y II, estuvo constituida por una caja Petri y se contó con cuatro repeticiones por cada tratamiento evaluado. Para la primera y segunda fase se estableció un total de 44 UE.

\section{Colecta del material vegetal}

Fueron colectadas hojas frescas de orégano (Oreganum vulgare) del municipio de Copainalá, Chiapas, frutos secos de pimienta (Pimienta dioica) de Comalcalco, Tabasco, hojas frescas de neem (Azadirachta indica) de Tuxtla 
Gutiérrez, Chiapas y hojas secas de matarratón (Gliricidia sepium) de la localidad Emiliano Zapata del municipio de Tecpatán, México. El material vegetal fue colectado en el mes de agosto del año 2018 y llevado al Laboratorio de Agrotecnologías de la Agencia Universitaria para el Desarrollo (AUDEs) Cacao-Chocolate de la Universidad Autónoma de Chiapas, para su selección y clasificación, evitando aquellas que presentaban afectaciones por insectos y enfermedades. Las hojas y frutos utilizados fueron secados en el invernadero y guardados en un lugar seco para evitar su humedecimiento y contaminación por insectos $\mathrm{u}$ hongos.

Obtención de los extractos vegetales

Los extractos vegetales fueron obtenidos mediante dos métodos de extracción, los cuales se describen a continuación:

Destilación: se usó la metodología descrita por Ramírez, et al., (2016), para lo cual se utilizó el material vegetal, bien picado o triturado y como disolvente agua destilada y alcohol etílico (9:1). Para obtener el extracto se empleó un destilador adaptado para este fin. El material vegetal se colocó dentro de la marmita del destilador junto con el disolvente, se tapó herméticamente para hacer el proceso de extracción continuo mediante la aplicación de calor y presión constante, el vapor se conducía a un condensador y mediante enfriamiento con agua corriente se obtenía el destilado.

Microondas: fue colocado el material vegetal triturado o en trozos pequeños en una bolsa de tela, que se sumergía en un vaso de precipitado con agua destilada estéril y alcohol etílico (9:1). Posteriormente se empleaba la radiofrecuencia de un microondas para la extracción de los metabolitos, bajo las siguientes condiciones: $220 \mathrm{KW}$ de potencia, un periodo de $150 \mathrm{y}$ 2.450 MHz (Ramírez, et al., 2016).

FASE 1. Efectividad antifúngica de extractos en una concentración al 60\% sobre C. gloeosporioides: Se prepararon cajas Petri con medio de cultivo Papa Dextrosa Agar (PDA), adicionado con el extracto al 60\% (v/v) y se realizó la inoculación del patógeno. Los cultivos fueron mantenidos bajo condiciones controladas (incubadora) de $23^{\circ} \mathrm{C} \pm 2{ }^{\circ} \mathrm{C}$. Se evaluaron ocho extractos vegetales utilizando sus dos formas de extracción (destilado y microondas) y tres controles; siendo PDA el control negativo y los controles positivos el fungicida de síntesis química (I.A. Ciprodinil 37,5\% + Fludioxonil 25\%) y el producto patente M2 UNACH. El efecto inhibitorio se cuantificó cada 24 horas durante 12 días mediante el crecimiento del diámetro del micelio. Se realizó un raspado superficial del hongo y un lavado con agua destilada y empleando la dilución apropiada. Se cuantificó la producción de esporas totales y las germinadas. 
FASE 2. Determinación de la concentración mínima inhibitoria sobre la cepa del hongo C. gloeosporioides.: los extractos que presentaron inhibición total del crecimiento y desarrollo del patógeno a la concentración del 60\% (v/v), fueron utilizados para los ensayos correspondientes a esta etapa, en la que se determinó la concentración mínima inhibitoria (CMI); estos productos fueron evaluados a concentraciones del 50\%, 40\%, 30\%, $20 \%$ y $10 \%(\mathrm{v} / \mathrm{v})$. En todas las pruebas se incluyó un control negativo (PDA).

Se preparó el medio de cultivo con PDA al cual se añadió cada uno de los extractos a las concentraciones a evaluar. Posteriormente se realizó la inoculación del patógeno. Como variable indicadora del efecto inhibitorio se midió cada 24 horas el diámetro de crecimiento del micelio del patógeno durante 12 días, también se cuantificó la producción de esporas totales y las germinadas mediante el uso de la cámara de conteo de esporas Neubauer, tal como se describió en la Fase 1.

El diseño experimental fue completamente al azar, para determinar los efectos de los tratamientos estudiados, se realizó un análisis de varianza (ANOVA) y en los casos en donde se encontraron diferencias significativas se aplicó la prueba de comparación de medias de Tukey $(\mathrm{P} \leq 0.05)$, usando el software SPSS versión 17.0 para Windows.

\section{RESULTADOS Y DISCUSIÓN}

FASE 1. Efectividad de extractos vegetales a una concentración del $60 \%$

Los resultados obtenidos se presentan en la Tabla 1 y Figura 1, donde se aprecia que los tratamientos de pimienta obtenida por microondas y el tratamiento de M2 UNACH fueron los únicos que no permitieron el crecimiento del patógeno. El ANOVA realizado indicó diferencias estadísticas entre los tratamientos para las variables crecimiento y número total de conidias. La prueba de medias de Tukey para la variable crecimiento registró diferencias significativas de pimienta obtenido por microondas y patente $\mathrm{M} 2$, con todos los tratamientos incluyendo el control positivo donde se utilizó el producto de síntesis química, el cual registró crecimiento, así como con el control negativo, pero éste no registró diferencias con los extractos obtenidos de matarratón y orégano, obtenidos por los dos métodos y con neem obtenido por destilación. 


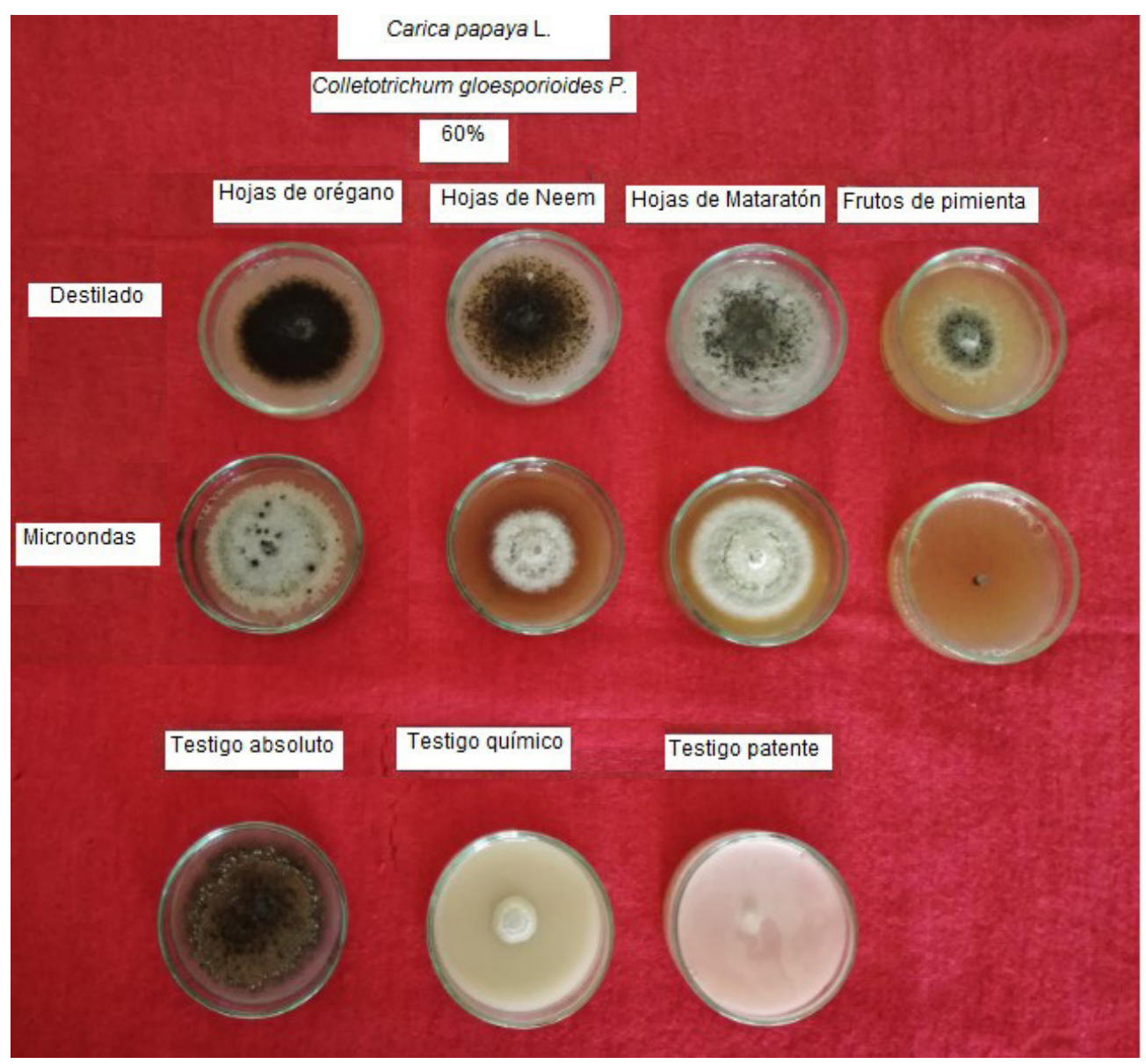

Figura 1. Efectos in vitro de extractos vegetales al $60 \%$ sobre Colletotrichum gloesporioides de C. papaya

Se observa que para el número de conidias totales, los extractos de orégano (destilado y microndas), neem obtenido por microondas, pimienta obtenido por destilación y el testigo químico a pesar que registraron crecimiento, redujeron la producción de conidias entre el 96.08 y el $99.9 \%$, mientras que tanto los tratamientos de pimienta obtenido por microondas y patente M2 no registraron formación de conidias, tratamientos que entre ellos no registraron diferencias estadísticas pero si con el control negativo y todos con el matarratón obtenido por destilación, el cual obtuvo el mayor valor superando al control negativo en $21.55 \%$, lo que indica que el extracto estimuló el desarrollo del patógeno, lo cual lo podría volver potencial como medio de cultivo en la obtención in vitro del patógeno para futuras investigaciones, al igual que Verastegui (1995), quien a partir de su investigación en el análisis del efecto antifúngico de 20 extractos de plantas llegó a la conclusión de que algunos aceites esenciales pueden inhibir el crecimiento, sin embargo otros lo pueden estimular. Así mismo, el testigo de producto de síntesis química presentó un leve crecimiento micelial de $13.75 \mathrm{~mm}$ y desarrollo de 
esporas, sin embargo, al ser observadas en el microscopio se encontraban deshidratadas o con afectaciones en su estructura física, como se observa en la Figura 2, lo que manifestó la acción del producto sobre el patógeno.

Con respecto al número de conidias germinadas, todos los tratamientos presentaron valores menores que los registrados por el control negativo, reduciendo entre el $10.86 \%$ y el 100\% la cantidad de éste tipo de estructuras; en los tratamientos de orégano y pimienta obtenidos por microondas y patente M2 UNACH, aún superaron la efectividad del control positivo producto de síntesis química, el cual permitió la germinación de esporas (1.04 x 103), mientras que éstos tres tratamientos la inhibieron completamente. En el caso de orégano microondas no se encontraron conidias germinadas, a pesar de que había crecimiento micelial y el número de conidias totales en su mayoría, al ser observadas presentaban daños en su estructura, lo que indica algún efecto antifúngico para C. gloesporioides, lo cual reafirma que es una planta con potencial fúngico que a su vez es corroborado con lo afirmado por Valverde (2017) quien referencia a Arcila, et al. (2004) y Tanackov (2013), los cuales indicaron que el aceite esencial de orégano tiene capacidad antimicótica contra varios microorganismos, entre los que se destacan a los siguientes: Cándida albicans, C. tropicalis, Torulopsis, Glabrata, Aspergillus níger, Geotrichum y Rhodotorula. Verastegui (1995) afirmó que Davidson (1993) realizó una revisión de las especies y saborizantes de alimentos con actividad antimicrobiana. De entre las que demostraron actividad antifúngica están la canela, el clavo, el orégano, el ajo y la cebolla. A su vez se estudió el mecanismo de acción del orégano y se encontró que produce alteraciones en el metabolismo microbiano particularmente en la respiración y la esporulación y se estableció que esto es debido a la disminución de la energía celular.

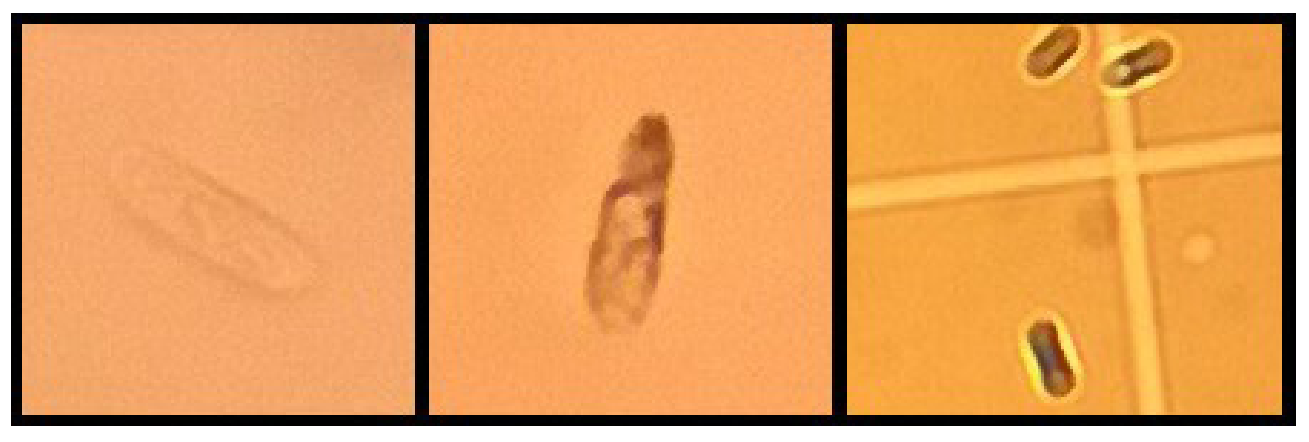

(A)

(B)

(C)

Figura 2. Efectos ejercidos por extracto de Oregano Microonda y I.A. Ciprodinil 37.5\% + Fludioxonil 25\% sobre C. gloesporioides aislado de C. papaya. A) espora normal, B) espora deshidratada por la exposicion al extracto vegetal C) espora hialina causa del efecto del I.A. Ciprodinil 37.5\% + Fludioxonil 25\% 
Los anteriores resultados corroboran las investigaciones de Joya, et al. (2017) y Tamayo, et al. (2016), quienes encontraron actividad antifúngica de orégano tanto en la reducción del crecimiento como en la formación y germinación de conidias de Moniliophthora roreri, además, la presente investigación muestra que es de alta importancia la forma de extracción utilizada, debido a que se puede expresar en mayores o menores cantidades los metabolitos secundarios y variar el porcentaje de inhibición fitopatógena, así mismo Ospina (2012) quien cita a Mahmoud, et al. (2011) documenta una disminución de 50\% de actividad antifúngica cuando se extrae el nimonol al extracto orgánico de hojas de neem en evaluaciones con hongos patógenos como Microsporum canis, Candida albicans y Aspergillus sp., con respecto a otros tipos de extracción evaluados. Así mismo Ramírez, et al. (2016) reportaron que la obtención de extractos asistidos por microondas permite obtener una mayor concentración de ellos, lo que logró reducir la cMi en la que afectan a los patógenos en comparación al método de destilación tradicional.

\section{Tabla 1}

Efectos in vitro de los extractos vegetales al 60\% sobre C. gloesporioides, aislado de C. papaya

\begin{tabular}{cccc}
\hline Tratamientos & $\begin{array}{c}\text { Crecimiento micelial } \\
(\mathrm{mm})\end{array}$ & $\begin{array}{c}\text { Conidias totales } \\
\left(\times 10^{4} \mathrm{~mL}^{-1}\right)\end{array}$ & $\begin{array}{c}\text { Conidias germinadas } \\
\left(\times 10^{3} \mathrm{~mL}^{-1}\right)\end{array}$ \\
Orégano Destilado & $47.75^{\mathrm{d}}$ & $41.14^{\mathrm{a}}$ & 1.04 \\
Orégano Microondas & $43.75^{\mathrm{d}}$ & $7.29^{\mathrm{a}}$ & 0 \\
Neem Destilado & $50.00^{\mathrm{d}}$ & $2080.72^{\mathrm{ab}}$ & 568.75 \\
Neem Microondas & $29.25^{\mathrm{c}}$ & $15.93^{\mathrm{a}}$ & 9.37 \\
Matarraton Destilado & $50.00^{\mathrm{d}}$ & $6261.87^{\mathrm{c}}$ & 1059.37 \\
Matarraton Microondas & $42.00^{\mathrm{d}}$ & $4.58^{\mathrm{a}}$ & 1.04 \\
Pimienta F Destilado & $40.25 \mathrm{c}^{\mathrm{d}}$ & $201.56^{\mathrm{a}}$ & 26.041 \\
Pimienta F Microondas & $0^{\mathrm{a}}$ & $0^{\mathrm{a}}$ & 0 \\
Control Positivo Patente M2 UNACH & $0^{\mathrm{a}}$ & $0^{\mathrm{a}}$ & 0 \\
Control Positivo (Químico) & $13.75^{\mathrm{b}}$ & $20.41^{\mathrm{a}}$ & 1.04 \\
Control negativo (Testigo absoluto) & $50.00^{\mathrm{d}}$ & $5151.66^{\mathrm{bc}}$ & 1188.54 \\
\hline
\end{tabular}

*Letras diferentes en columnas presentan diferencias significativas (Tukey, $\mathrm{P} \leq 0.05$ )

FASE 2. Determinación de la concentración mínima inhibitoria (CMI)

Para la determinación de la concentración mínima inhibitoria se tuvieron en cuenta aquellos tratamientos que inhibieron completamente el desarrollo del patógeno a una concentración del $60 \%$, posteriormente se evaluaron concentraciones del 50, 40, 30, 20 y 10\% (V:V) sobre C. gloeosporioides aislado de papaya, estos tratamientos fueron los correspondientes al extracto de 
fruto de pimienta y patente M2 UNACH en la fase 1, los resultados obtenidos en esta fase se pueden apreciar en la Figura 3 y la Tabla 2, a partir de la realización del ensayo se encontró que hay un efecto de inhibición fúngica de los dos productos, que se manifiesta de acuerdo a la concentración para la variable de crecimiento micelial se observó que a medida que disminuía la concentración del extracto vegetal o de la patente aumentaba el diámetro del micelio de forma más acelerada respectivamente, acercándose consecutivamente al control negativo, a su vez se manifestaba un cambio en la coloración del micelio con los productos utilizados, pues a medida que disminuía la concentración de los productos se observaba un color más oscuro en el micelio, lo que se relaciona directamente con la esporulación del patógeno, es decir con la variable número de esporas.

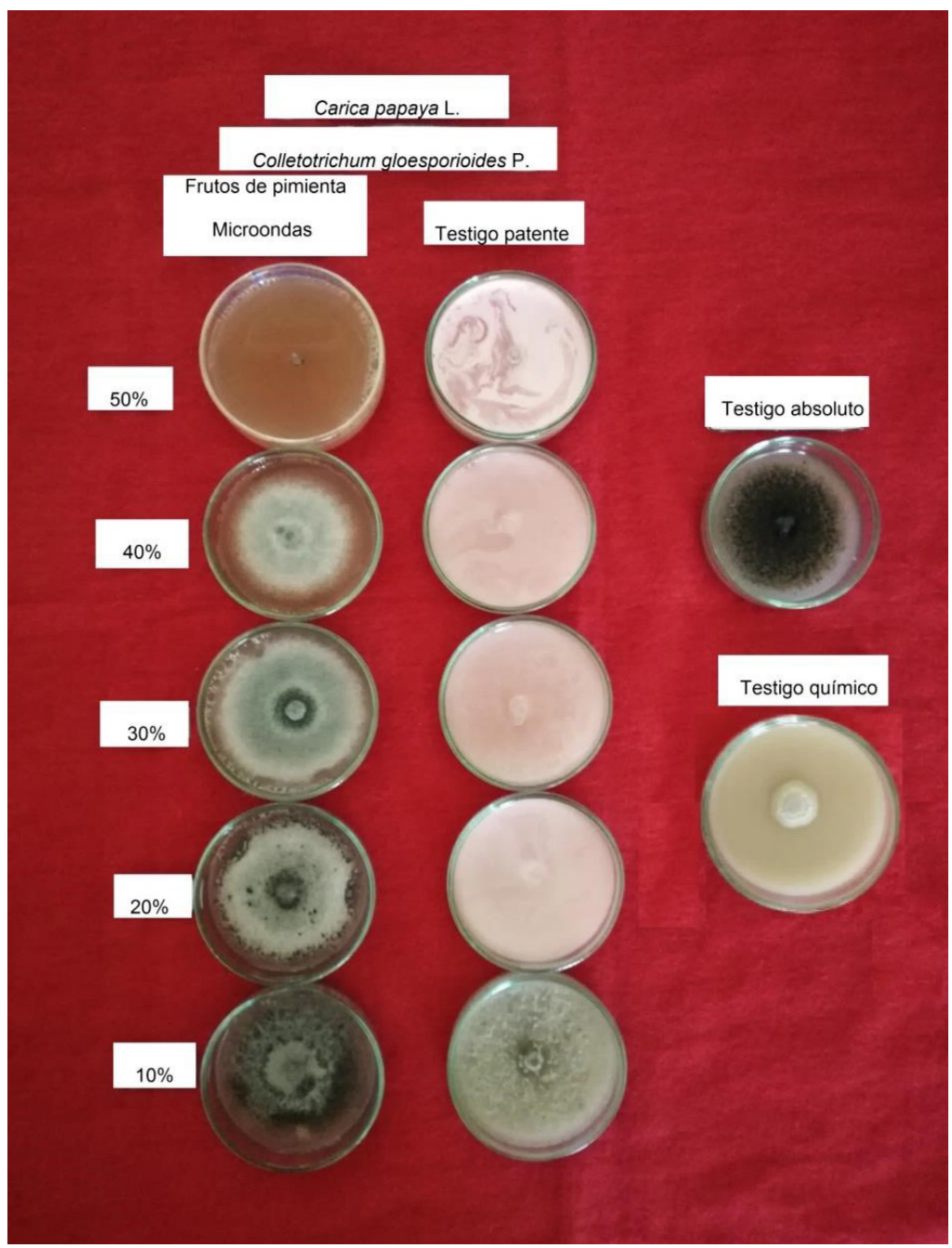

Figura 3. Efectos de del extracto de fruto de pimienta y patente M2 UNACH al 50\%, 40\%, 30\%, 20\% y 10\% sobre Colletotrichum gloesporioides de C. papaya 
Al determinar la CMI para la patente M2 UNACH, se encontró que inhibió el patógeno hasta una concentración del $20 \%$, a su vez presentando diferencias significativas con respecto a la concentración del 10\% y el control negativo, según la prueba de medias de Tukey $(\mathrm{P} \leq 0.05)$, como se muestra en la Tabla 2. En cuanto a la CMI, para el extracto de fruto de pimienta obtenido por microondas sobre C. gloesporioides, se encontró que únicamente inhibió completamente a una concentración del 50\%, a partir del $40 \%$ se observó crecimiento micelial acercándose progresivamente al control negativo, así mismo, en cuanto a la formación de esporas, a partir del $40 \%$ fue aumentando, siendo estadísticamente diferente al 20\%, y este respectivamente diferente al control negativo, estos resultados obtenidos difieren con respecto a los presentados por Jiménez (2018) quien afirmó que el extracto de pimienta fruto presentó inhibición parcial del $21.34 \%$ sobre el crecimiento radial del mismo hongo, mientras en conidias totales y germinadas registraron del 66.80 al $91.49 \%$ de inhibición respectivamente. A su vez se asemejan a los resultados presentados por Ramírez, et al. (2016) quienes exponen que los extractos del fruto de pimienta obtenidos mediante el método de destilado y asistido por microondas inhibieron in vitro a $C$. gloeosporioides aislado de papaya a concentraciones del 30 y $20 \%$.

A pesar de que se observó producción de esporas, a medida que aumentaba la concentración iba presentando inhibición del patógeno progresivamente, lo que hace que el producto patente M2 UNACH y al extracto vegetal de fruto de pimienta obtenido por microondas, sean productos potencialmente efectivos para el control del patógeno en la concentración adecuada. Así mismo en los tratamientos que presentaron crecimiento micelial registraron formación de conidias y germinadas, las cuales mostraron diferencias estadísticas con respecto al testigo, inhibiendo la formación de conidias entre el 47.09 y $100 \%$ y la germinación entre el 72.54 y 100\%, además, en observaciones realizadas en el microscopio, se apreció que en los tratamientos con concentraciones altas de producto se observaban conidias con afectaciones en su estructura o forma.

Barros, et al., (2020), identifica mediante cromatografía de gases acoplada a espectrometría de masas (GC-EM) los compuestos del aceite de $P$. dioica obtenido mediante hidrodestilación, obteniendo que contenía $76,88 \%$ de eugenol, mismos que inhibieron el desarrollo micelial de hongos hasta 97,78\%.

Padrón (2010) reporta que en general, los aceites esenciales poseen fuertes propiedades antibacterianas debido a que contienen un alto porcentaje de compuestos fenólicos como el eugenol. Lo anterior sugiere que su mecanismo de acción sea similar al de otros compuestos fenólicos por alteración de la membrana citoplasmática, interrumpiendo la fuerza motriz de protones (PMF), el flujo de electrones, el transporte activo y la coagulación del contenido celular. 
Se ha encontrado que a concentraciones subletales de eugenol se inhibe la producción de amilasa y proteasas de B. cereus, deteriorando la pared celular, lo que origina la lisis celular. Se cree que el grupo hidroxilo del eugenol al que se unen ciertas proteínas, previene la acción enzimática en E. aerogenes (Denyer y Hugo, 1991b; Sikkema, et al., 1995.; Davidson, 1997; Wendakoon y Sakaguchi, 1995 citados por Padrón), por lo que posiblemente la acción mostrada por el extracto obtenido mediante microondas de $P$. dioica sobre C. gloesporioides aislado de papaya se deba a este compuesto.

\section{Tabla 2}

Determinación in vitro de la CMI de pimienta fruto y Patente M2 UNACH sobre el crecimiento y desarrollo de Colletotrichum gloesporioides aislado de C. papaya

\begin{tabular}{cccc}
\hline Tratamientos & $\begin{array}{c}\text { Crecimiento micelial } \\
(\mathrm{mm})\end{array}$ & $\begin{array}{c}\text { Conidias totales } \\
\left(\times 10^{4} \mathrm{~mL}^{1}\right)\end{array}$ & $\begin{array}{c}\text { Conidias germinadas } \\
\left(\times 10^{3} \mathrm{~mL}^{-1}\right)\end{array}$ \\
T. patente $50 \%$ & $0^{\mathrm{a}}$ & $0^{\mathrm{a}}$ & $0^{\mathrm{a}}$ \\
T. patente $40 \%$ & $0^{\mathrm{a}}$ & $0^{\mathrm{a}}$ & $0^{\mathrm{a}}$ \\
T. patente $30 \%$ & $0^{\mathrm{a}}$ & $0^{\mathrm{a}}$ & $0^{\mathrm{a}}$ \\
T. patente $\mathbf{2 0 \%}$ & $0^{\mathrm{a}}$ & $0^{\mathrm{a}}$ & $0^{\mathrm{a}}$ \\
T. patente $\mathbf{1 0 \%}$ & $50.00^{\mathrm{c}}$ & $70.52^{\mathrm{a}}$ & $6.25^{\mathrm{a}}$ \\
Pimienta F Micr 50\% & $0^{\mathrm{a}}$ & $0^{\mathrm{a}}$ & $0^{\mathrm{a}}$ \\
Pimienta F Micr 40\% & $42.50^{\mathrm{b}}$ & $524.58^{\mathrm{ab}}$ & $34.37^{\mathrm{a}}$ \\
Pimienta F Micr 30\% & $50.00^{\mathrm{c}}$ & $910.52^{\mathrm{abc}}$ & $16.35^{\mathrm{a}}$ \\
Pimienta F Micr 20\% & $50.00^{\mathrm{c}}$ & $3636.61^{\mathrm{c}}$ & $76.4^{\mathrm{a}}$ \\
Pimienta F Micr 10\% & $50.00^{\mathrm{c}}$ & $3088.22^{\mathrm{bc}}$ & $92.18^{\mathrm{a}}$ \\
Control negativo & $50.00^{\mathrm{c}}$ & $6873.43^{\mathrm{d}}$ & $335.72^{\mathrm{b}}$ \\
\hline
\end{tabular}

*Letras diferentes en columnas presentan diferencias significativas (Tukey, $\mathrm{P} \leq 0.05$ )

\section{CONCLUSIONES}

El extracto obtenido de frutos de Pimienta dioica por el método de microondas a una concentración del $50 \% \mathrm{v} / \mathrm{v}$ resulta ser efectivo en el control in vitro de C. gloesporioides asilado de Carica papaya.

La forma de extracción por microondas de A. indica, O. vulgare, G. sepium y $P$. dioica resultó ser más eficiente que por destilación, ya que a la misma concentración inhibieron el crecimiento, y la formación y germinación de conidias de C. glosporioides aislado de C. papaya.

El extracto obtenido por microondas de $P$. dioica y el producto de patente M2 UNACH, tienen potencial de ser una alternativa para el control de la antracnosis de papaya, al reducir el crecimiento y desarrollo del patógeno. 


\section{BIBLIOGRAFÍA}

Barros, P. R., Da Silva, F. R., Batista J., Oliveira, G., Santos de Oliveira, G., Wesly, R., Costa, H, Alves, M., Crispim, A., Do Livramento, M. y Mouchrek, V. E. (2020). Chemical composition and biological activity of the essential oil of the fruits Pimenta dioica against formae speciales of fungus Fusarium oxysporum. Rev. Colomb. Cienc. Quím. Farm. 49(1), 89-100. DOI: https://doi.org/10.15446/rcciquifa.v49n1.87010

Corporación Colombiana de Investigaciones Agropecurias. (2000). El cultivo de la papaya en los llanos orientales de Colombia. Manual de asistencia Técnica $N^{\circ} 4$. Villavicencio. Colombia.

Departamento Nacional de Estadística. (2016). El cultivo de la papaya (Carica papaya L.) y sus principales enfermedades en época de lluvias. Boletín mensual insumos y factores asociados a la producción agropecuaria; $\mathrm{N}^{\circ} 47$.

Dirzo, R. (1985). Metabolitos secundarios en las plantas. Atributos panglossianos o de valor adaptativo. Ciencia. 36, 137:145.

Echeverría, L. (2012). Efectividad biológica del fungicida Azoxystrobin en Alternaria alternata de tomate (Solanum lycopersicum L.) in vitro. [Tesis Ingeniero Agrónomo, Universidad Autónoma Agraria Antonio Narro] Repositorio Institucional. http://repositorio.uaaan.mx

Evans, E. (2015). Una mirada a la producción, el comercio y el consumo de papaya a nivel mundial. University of Florida.

Hernández, A., Bautista, S., Velásquez, S. (2007). Prospectiva de extractos vegetales para controlar enfermedades postcosecha hortofrutícolas. Revista fitotécnia Mexicana, 30(2), 119-123.

Instituto de Investigaciones Agropecuarias. (2016). Ministerio de Agricultura. Instituto de Investigaciones Agropecuarias. Villa Alegre. https://www.intagri.com/articulos/fitosanidad/manejo-de-la-antracnosisen-el-cultivo-de-papaya

Jiménez, S. (2018). Extractos de plantas y preparados minerales sobre Colletotrichum gloeosporioides, Phytophthora spp. y Alternaria alternata. [Tesis de Maestría en Ciencias en Producción Agropecuaria Tropical, Universidad Autónoma de Chiapas]. Repositorio Institucional Universidad Autónoma de Chiapas.

Joya, G., Alvarado, E., Ramirez, S. y López, O. (2017). Optimización de la destilación de Origanum vulgare L., con efecto antifúngico en Moniliophthora roreri (cif \& par); Espacio I + D. 6(14).138-151. doi: 10.31644/IMASD.14.2017.a07

Martínez, A., Contreras, J., Belares, R. (2010). Extracción de polifenoles asistida por microondas a partir de Punica granatum L. Revista Científica de la Universidad Autónoma de Coahuila, 2(4). http:// https://docpla- 
yer.es/72568736-Extraccion-de-polifenoles-asistida-por-microondas-a -partir-de-punica-granatum-1.html

Montaño, A. (2015). Estudio de la incidencia de infecciones quiescentes de Colletotrichum spp., en flores y frutos de mora (Rubus glaucus). [Tesis de Ingeniería Agronómica. Universidad de Cundinamarca] http:// https:// docplayer.es/26998530-Estudio-de-la-incidencia-de-infecciones-quiescentes-de-colletotrichum-spp-en-flores-y-frutos-de-mora-rubus-glaucus-benth.html

Ospina, D. (2012). Actividad antifúngica del extracto crudo de Azadirachta indica a. Juss. de suspensión de células sobre hongos dermatofitos causantes de enfermedades patógenas al hombre. [Tesis de maestría en Ciencias Agrarias. Universidad Nacional de Colombia] Repositorio Institucional http:// http://bdigital.unal.edu.co/8083/1/14624562.2012.pdf

Padrón, M.B. 2010. Componentes químicos con actividad bactericida, fungicida $y$ citotóxica de plantas de la familia Myrtaceae y Lauraceae. [Tesis Doctorado; Universidad Autónoma de Nuevo León, México]. Repositorio institucional http://cdigital.dgb.uanl.mx/te/1080211163.PDF

Parra, L. (2008). Relación entre infecciones quiescentes de Colletotrichum gloesporioides (Penz) y los diferentes estados fenológicos del fruto de mango (Magnifera indica L.) variedad hilacha. [Tesis de grado de Microbiología agrícola y veterinaria. Pontificia Universidad Javeriana]. Repositorio Institucional https:// www.javeriana.edu.co/biblos/tesis/ciencias/tesis120.pdf

Pérez, N. y Jiménez, E. (2011). Producción de metabolitos secundarios de plantas mediante el cultivo in Vitro. Biotecnología vegetal. 11(4), 195 - 211.

Puertas, M., Ríos, Y. y Rojano, B.; (2013). Determinación de antocianinas mediante extracción asistida por radiación de microondas en frijol (Phaseolus vulgaris L.) de alto consumo de Antioquia. Revista cubana de plantas medicinales, 18(2), 288-297. http://www.revplantasmedicinales. sld.cu/index.php/pla/article/view/50

Quercuslab. (2015). Destilación simple y a vacío. https://quercuslab.es/blog/ destilacion-simple-y-a-vacio/

Quiroga. (2016). Antracnosis, una enfermedad limitante para la producción de papaya. APPAlsi (Asociación Agroecológica de Productores y Comercializadores de Papaya del Alto Sinú). http://www.croplifela. org/es/proteccion-de-cultivos/plaga-del-mes/antracnosis-papaya.html

Ramírez, S; López, O.; Espinosa, S.; Wong, A. (2016). Actividad antifúngica de hidrodestilados y aceites sobre Alternaria solani, Fusarium oxysporum, Colletotrichum gloeosporioides P. Revista Mexicana de Ciencias Agrícolas, $7(8), 1879-1891$.

Rodríguez, S.; González, P.; Mayek, N. (2009). La Infección de Colletotrichum gloeosporioides (Penz.) Penz. y Sacc. en Aguacatero (Persea americana 
Mill.): Aspectos Bioquímicos y Genéticos. Revista mexicana de fitopatología, 27(1), 53-63.

Rojas, Alberto (2011). Manual de microbiología. Conceptos y práctica de microbiología general. Universidad Nacional de Colombia, Sede Palmira. 41-42.

Tamayo L., Ramírez S., López O., Quiroga R., Espinosa S. (2016). Extractos por destilación de Origanum vulgare, Tradescantia spathacea y Zingiber officinale para el manejo de Moniliophthora roreri de Theobroma cacao. Revista Mexicana de Ciencias Agrícolas, 7(5), 1065-1076.

Valverde, Q. (2017). Efectividad antimicótica del aceite esencial de orégano de las provincias de Chimborazo y Santa Elena al 100\% de concentración sobre Candida albicans. Universidad Central de Ecuador; Proyecto de investigación presentado como requisito previo a la obtención del título Odontólogo; Quito.

Ventura, J., Costa, H., Silva, J. (2004). Papaya Diseases and Integrated Control; S.A.M.H. Naqvi (ed.), Diseases of Fruits and Vegetables, Vol. II, 201-268, Kluwer Academic Publishers. Printed in the Netherlands.

Verastegui, M. (1995). Análisis del efecto antifúngico de 20 extractos de plantas. Universidad Autónoma de Nuevo León. Tesis presentada como requisito parcial para obtener el grado de Maestro en Ciencias con Especialidad en microbiología; Monterrey; México.

Zavala, L., Suarez, J., Cristóbal, A., Ruiz, E., Gutiérrez, A., Calderón, V., Méndez, R. (2011). Control postcosecha de la antracnosis en papaya y sensibilidad de Colletotrichum gloeosporioides (Penz.) Sacc. a fungicidas organosintéticos. Revista Chapingo Serie Horticultura 11. 